\title{
SIPPs, Why Do We Need a New Standard for Interconnect Process Parameters?
}

\author{
Dr Martin G. Walker, Dr Keh-Jeng (KJ) Chang, Dr Christophe J. Bianchi \\ Frequency Technology, Inc., Technology Station, 469 El Camino Real, Santa Clara, CA \\ 95050 (408) 961-2300 (main line), (408) 961-2323 (FAX), christophe@frequency.com \\ (principal contact)
}

Key Words: SIPPs, interconnect, characterisation, process, extraction, modelling

Abstract:

The overall performance of deep sub-micron integrated circuits is dominated by the interconnect. Standard Interconnect Process Parameters (SIPPs) [1] provides an abstract model of the interconnect system, as well as a methodology to derive interconnects electrical parameters from a process description.

SIPPs also enables measurement of process variations and their impact on interconnect performance.

This paper describes in details the SIPPs model, as well as highlights some of the benefits of using a standard for interconnect parameters.

SIPPs is now an SI2 (Silicon Integration Initiative) [2] endorsed standardisation effort.

\section{INTRODUCTION}

The overall performance of deep sub-micron integrated circuits is dominated by the interconnect. One of the most difficult challenges of producing new, complex chips is providing process experts with tools to communicate the capabilities of their technology to designers. These two communities quite often use different terminologies. The adoption of SIPPs will bridge this gap and should result in higher performance devices coming out in shorter periods of time. Momentum is building among industry leaders for a program that can reduce or eliminate tool-specific interconnect 
characterization and provide highly accurate performance estimates as foundries implement very deep sub-micron processes.

Silicon Integration Initiative (Si2) has taken over leadership of the SIPPs development effort and is committed to going through an assessment of the technology. Si2 expects to complete SIPPs standardization within one year.

\subsection{Definition of SIPPs}

A set of parameters has been proposed that predicts the electrical performance of on-chip interconnect for multilevel-metal, VLSI processes. The proposed parameters ( SIPPs ) are a minimal set of parameters needed to accurately predict the resistance and capacitance of the state-of-the-art interconnect systems. SIPPs describe a simple physical model, so they can be used as a common interconnect parameter set for all design calculations, including $\mathrm{R} / \mathrm{C}$ extraction tools.

The proposed SIPPs are based on a model for the interconnect system. The cross-section of the model, shown in Figure 1, comprises: rectangular polygons representing conductors, planar dielectrics filling the volumes between them, and cylindrical vias (not shown).
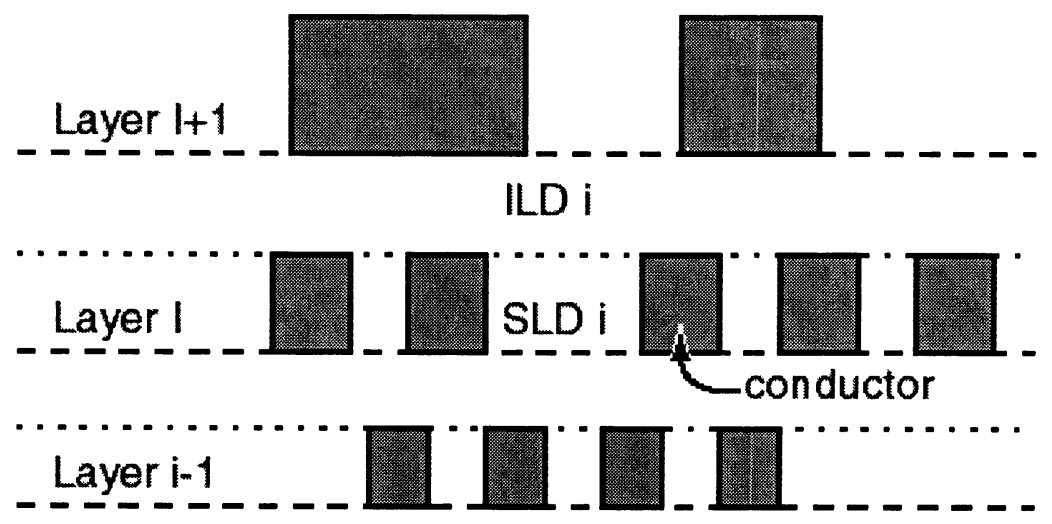

Figure 1. Cross section of a SIPPs model

For each interconnect layer, there are two planar dielectrics: one lies between the conducting lines on the same layer (same-layer dielectric or SLD), and the other is between the conductors of a layer and the layer above (inter-layer dielectric or ILD). The dielectric constants of the two need not to be the same. A via is modeled as a cylinder connecting two conductor polygons at adjacent layers. The height of the via is the thickness for ILD.

SIPPs are electrically predictive parameters, not an exact replica of interconnect geometry. The model closely approximates, but does not exactly replicate, the interconnect geometry of current IC processes. Instead, 
it intentionally simplifies the actual physical geometry. Nonetheless, the model provides an electrically equivalent model for the interconnect system when dimensioned with properly chosen SIPPs. It should accurately predict the electrical performance of the interconnect system. For example, using a properly dimensioned model, a field solver operating on suitable test structures will predict Rs or Cs whose values match those measured from silicon.

As an example of a typical simplification, fabricated aluminum conductors often consist of a sandwich of barrier metal surrounding an aluminum core. The cross section of such a wire may look I-shaped, due to different etch rates between aluminum and the barrier metal, or trapezoidal, due to different etch rates at different depths. The model accounts for all of these effects with equivalent rectangular polygons that predict wire resistance and capacitance as accurately as possible.

Similarly, a complex multi-material dielectric is usually deposited between two metal levels. It is not represented explicitly in the SIPPs model, but is summarized by the planar dielectrics and the conductor polygons.

\section{SIPPs}

\subsection{Description for each parameter in SIPPs}

For each interconnect layer in an interconnect system, the SIPPs based on the above model are shown in Figure 2.

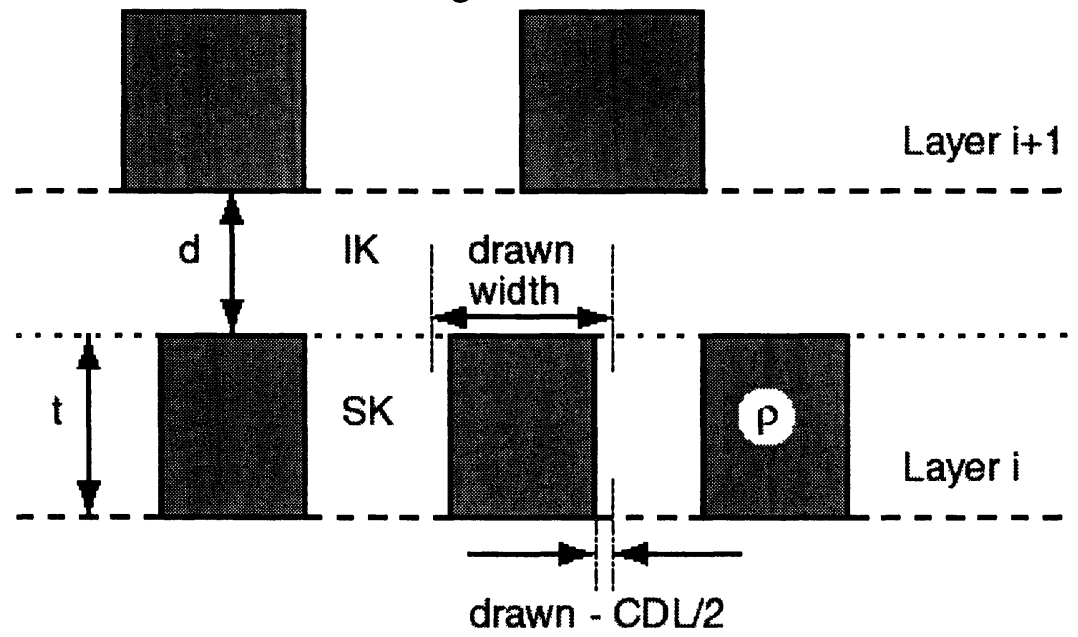

Figure 2. SIPPs associated with a layer. 
1. Sheet resistance $(\rho)$ : is the sheet resistance of the conductor layer. For example, sheet resistance for Metal 2 may be $0.05 \Omega$ /square. Sheet resistance is a piece-wise linear function of drawn width.

2. Critical dimension loss (CDL): is the loss in the conductor width compared with drawn width. For example, the actual width of a Metal 2 wire of $0.5 \mu \mathrm{m}$ drawn width and $0.1 \mu \mathrm{m} \mathrm{CDL}$ is $0.4 \mu \mathrm{m}$. CDL is a twodimensional, piece-wise linear function of drawn width and drawn spacing.

3. Conductor thickness (t): is the thickness of the rectangular conductor polygons in the model. For example, conductor thickness for Metal 2 may be $0.6 \mu \mathrm{m}$.

4. Dielectric constant for same-layer dielectric (SK): is the dielectric constant for same-layer dielectric (SLD), the planar dielectric between conductor polygons of the same layer. For example, SK may be 3.9 for the dielectric between Metal 2 polygons. SK is a piece-wise linear function of drawn spacing.

5. Dielectric constant for inter-layer dielectric (IK): is the dielectric constant for inter-layer dielectric (ILD), the planar dielectric between conductor polygons of this interconnect layer and the layer above. For example, IK may be 4.1 for the dielectric between Metal 2 and Metal 3 layers.

6. Inter-layer dielectric thickness (d): is the distance between conductor polygons of this interconnect layer and the layer above. For example, $d$ may be $0.8 \mu \mathrm{m}$ between Metal 2 and Metal 3 layers. $\mathrm{d}$ is a twodimensional, piece-wise linear function of drawn width and drawn spacing of the underlying conductor polygons.

7. Via resistance $\left(R_{\text {via }}\right)$ and via diameter $\left(\mathbf{d}_{\text {via }}\right)$ : are the resistance and diameter of the via cylinder. For example, $R_{\text {via }}$ may be $3 \Omega$ and $d_{\text {via }}$ may be $0.5 \mu \mathrm{m}$ for Metal 2 ñ Metal 3 vias.

\subsection{Description of how to incorporate variations into SIPPs}

Instead of a single number, each parameter in a SIPPs set is defined by a pair of numbers: mean and standard deviation. SIPPs assume that the probability distribution of each parameter is Gaussian. Thus, SIPPs represent process variations, and they allow you to determine performance corners for the interconnect.

Note that SIPPs do not include basic design rules, such as minimal width and spacing for conductors. However, these design rules would ordinarily accompany the SIPPs to provide a complete picture of the interconnect. 


\section{USING SIPPS}

The SIPPs described here are used to accurately predict resistance and capacitance of the interconnect system from layout. Simple situations may require only hand calculations; complex ones generally require field solvers, such as Raphael $^{\mathrm{TM}}$, or QuickCap ${ }^{\mathrm{TM}}$ or other software.

$\begin{array}{llllll}\text { SIPP } & \text { Substrate } & \text { Poly } & \text { Metal1 } & \text { Metal2 } & \text { Passivation } \\ \mathrm{d} & 0.3 \pm 0.5 \% & 0.5 \pm 10 \% & 0.8 \pm 10 \% & 2.0 \pm 5 \% & 2.0 \pm 5 \% \\ \mathrm{IK} & 3.9 \pm 1 \% & 3.9 \pm 1 \% & 3.9 \pm 1 \% & 3.9 \pm 1 \% & 4.0 \pm 1 \% \\ \mathrm{t} & & 2.0 \pm 0.2 & 0.6 \pm 0.05 & 0.6 \pm 2 \% & \\ \text { SK } & & 3.9 \pm 1 \% & 4.1 \pm 1 \% & 4.1 \pm 1 \% & \\ \text { CDL } & & 0.05 \pm 0.5 \% & 0.1 \pm 0.02 & 0.1 \pm 2 \% & \end{array}$

Figure 3. SIPPs for the 2M1P example process

Figure 3 shows the SIPPs for a hypothetical 2M1P process, i.e., a process with two metal and one poly layers. For this process, constants adequately model all SIPPs, without need for piece-wise linear functional descriptions.

\subsection{Example: using SIPPs for predicting resistance}

Considering calculating the resistance of a Metal 2 wire of $200 \mu \mathrm{m}$ drawn length and $0.5 \mu \mathrm{m}$ drawn width for this example interconnect system. Metal 2 has a sheet resistance of $0.05 \Omega / 0$ and a CDL of $0.1 \mu \mathrm{m}$. Therefore, the wire will have resistance of:

$200 \mu \mathrm{m} /(0.5 \mu \mathrm{m}-0.1 \mu \mathrm{m}) * 0.05 \mathrm{~W} / \mathrm{o}=25 \Omega$.

Similarly, if CDL were 0 , the final line width on the silicon equals the $0.5 \mu \mathrm{m}$ drawn width, and the resistance drops to $20 \Omega$.

\subsection{Example: using SIPPs for predicting capacitance}

Figure 4 shows a structure consisting of 3 parallel Metal 2 wires running over a regular array of Metal 1 wires. Consider calculating the capacitance the middle Metal 2 wire per pitch of the underlying Metal 1 wire. Assuming widths and spacings of $0.5 \mu \mathrm{m}$ and $1.0 \mu \mathrm{m}$ for both the Metal 1 and the Metal 2 wires.

Accurate calculation of this capacitance requires a field solver simulation. All off the SIPPs listed in Table 1 except $\mathrm{CDL}_{\mathrm{p}}$ affect the capacitance. Appendix A shows the Raphael ${ }^{\mathrm{TM}}$ [3] input and output for an appropriate field solution for the structure. The desired capacitance is $0.19 \mathrm{fF}$ per Metal 1 pitch, or equivalently, $0.13 \mathrm{fF} / \mu \mathrm{m}$. 

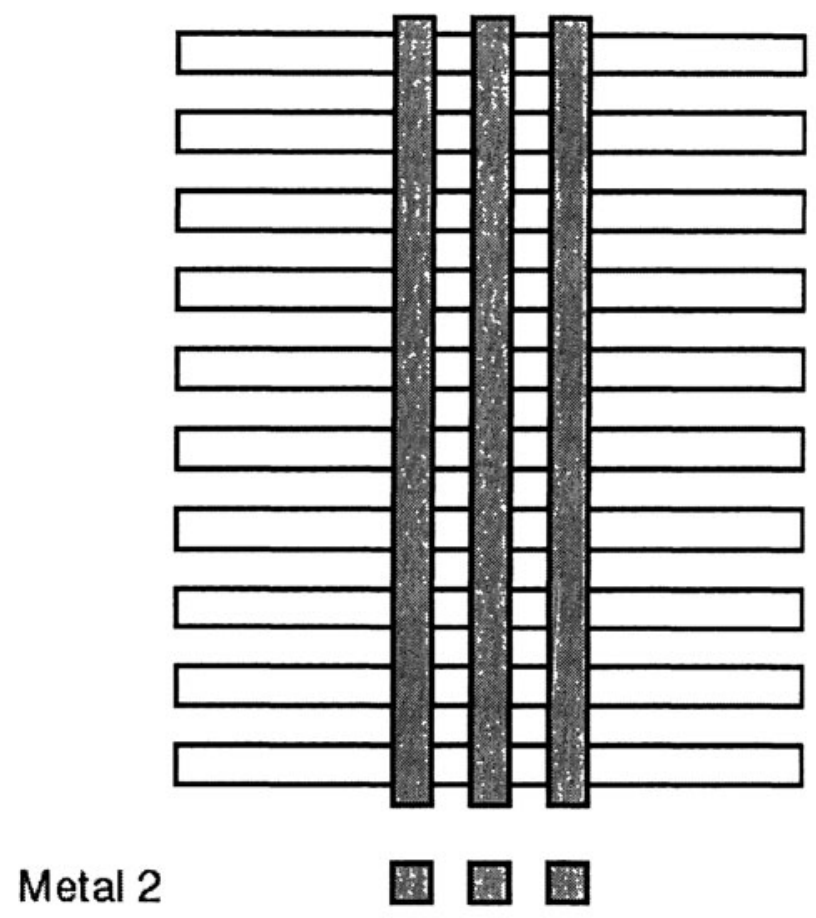

\section{Metal 1}

Figure 4. Structure for capacitance calculation, in top view and cross section.

Three Metal 2 wires cross above an array of ten Metal 1 wires. Determine the total capacitance of the center Metal 2 wire.

\subsection{Characterisation of SIPPs parameters}

SIPPs parameters can be verified on silicon by characterisation of test structures, electrical measurements, SEM measurements and end-to-end electrical performance checking [5].

By verifying SIPPs parameters, process-induced effects can be more accurately mapped into the design flow, allowing better modelling of RC parasitics [6]. 


\section{APPENDIX A. RAPHAEL EXAMPLE FOR CAPACITANCE}

This appendix contains the input and output for one possible Raphael [3] simulation of the cross-over structure of Figure 4 . In the code, " 0 " refers to everything below Metal 1, "1" refers to the Metal 1 layer, "2" refers to the Metal 2 layer, and "3" refers to the passivation above the Metal 2 layer. The total capacitance for M2line2, the center Metal 2 line, is $1.9 \mathrm{fF}$, so its capacitance per Metal 1 pitch is $0.19 \mathrm{fF}$.

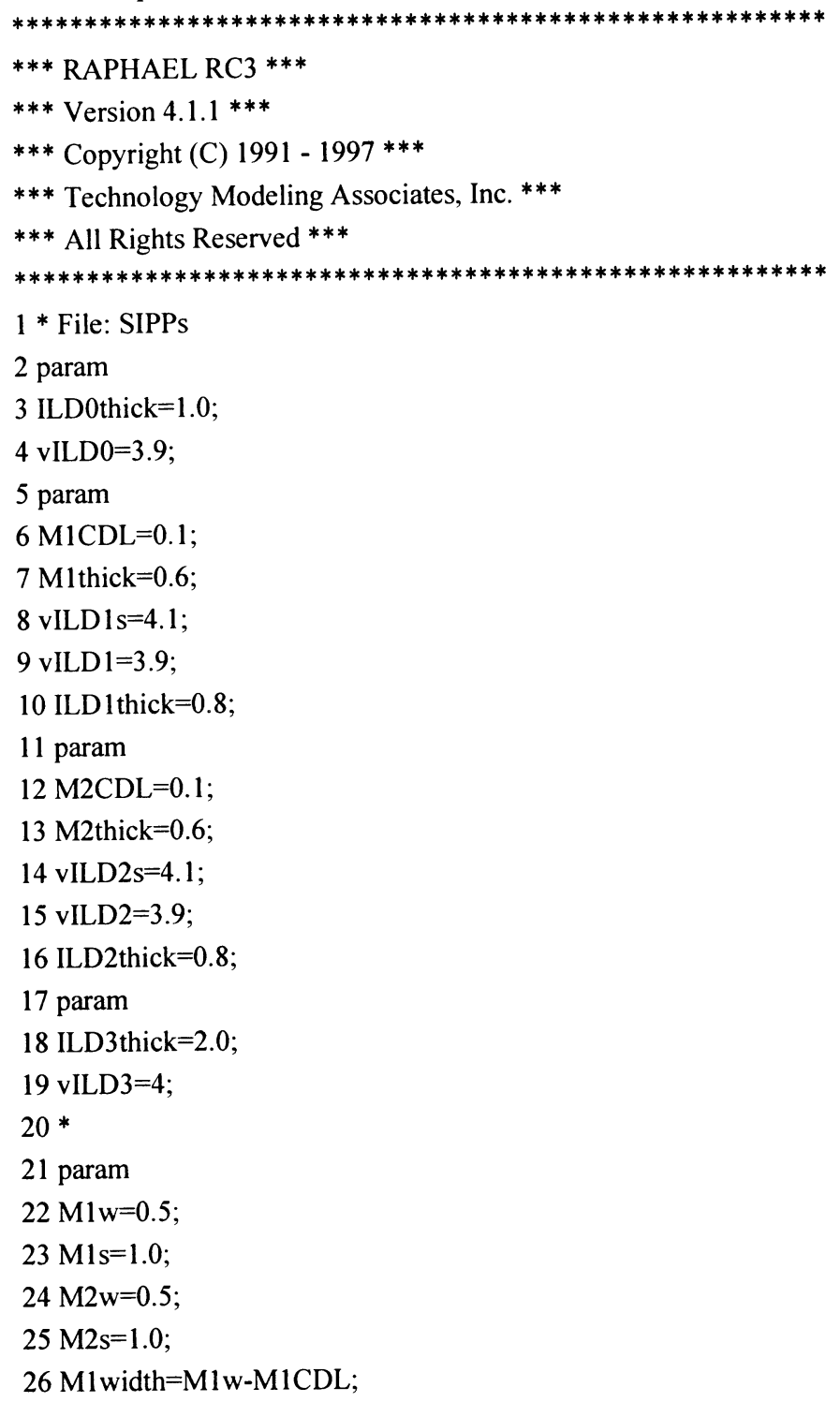


$27 \mathrm{M} 1$ space $=\mathrm{M} 1 \mathrm{~s}+\mathrm{M} 1 \mathrm{CDL}$;

$28 \mathrm{M} 2$ width=M2w-M2CDL;

$29 \mathrm{M} 2$ space $=\mathrm{M} 2 \mathrm{~s}+\mathrm{M} 2 \mathrm{CDL}$;

30 Sthick $=0.9$;

$31 *$

32 param

$33 \mathrm{M} 1$ pitch=M1 width+M1 space;

34 M2pitch=M2width+M2space;

35 Xtotal=10*M1 pitch;

36 Ytotal=10*M2pitch;

37 Ztotal=Sthick+ILD0thick+M1 thick+ILD0thick+M2thick+ILD2thick+ILD3thick;

38

39 block name=ILD0; width $=$ Xtotal; length $=$ Ytotal; diel=vILD0;

$40 \mathrm{v} 1=0,0$, Sthick;

41 v2 =0,0,Sthick+ILD0thick;

42

43 block name=ILD1s; width=Xtotal; length $=$ Ytotal; diel=vILD1s;

$44 \mathrm{vl}=0,0$, Sthick+ILD0thick;

45 v2 $=0,0$, Sthick+ILD0thick+M1 thick;

46

47 block name=ILD1; width=Xtotal; length=Ytotal; diel=vILD1;

48 v1 $=0,0$, Sthick + ILD0thick + M1 thick;

49 v2 $=0,0$, Sthick+ILD0thick+M1 thick+ILD1 thick;

50

51 block name=ILD2s; width=Xtotal; length=Ytotal; diel=vILD2s;

52 v1 $=0,0$, Sthick+ILD0thick+M1 thick+ILD1 thick;

53 v2 $=0,0$, Sthick+ILD0thick+M1 thick+ILD1 thick+M2thick;

54

55 block name=ILD2; width=Xtotal; length=Ytotal; diel=vILD2;

$56 \mathrm{vl}=0,0$, Sthick+ILD0thick+M1thick+ILD1 thick+M2thick;

$57 \mathrm{v} 2=0,0$, Sthick+ILD0thick+M1 thick+ILD 1 thick+M2thick+ILD2thick;

58

59 block name=ILD3lanket; width=Xtotal; length $=$ Ytotal; diel=vILD3;

$60 \mathrm{vl}=0,0$, Sthick+ILD0thick+M1 thick+ILD1thick+M2thick+ILD2thick;

61 v2=0,0,Sthick+ILD0thick+M1thick+ILD1thick+M2thick+ILD2thick+ILD3thick;

62

63 block name=M1line 10; width $=\mathrm{M} 1$ width; length $=$ Ytotal; volt $=0$;

$64 \mathrm{v1}=4.5^{*}$ M1 pitch,0,Sthick+ILD0thick;

65 v2=4.5*M1 pitch,0,Sthick+ILD0thick+M1 thick;

66

67 copy $3 \mathrm{~d}$ from=M1line $10 ;$ to $=\mathrm{M} 1$ line $9 ;$ direction= $=1 * \mathrm{M} 1$ pitch, 0,$0 ;$ volt $=0$; 
69 copy $3 \mathrm{~d}$ from=M1line9; to=M1line8; direction=-1*M1pitch, 0,0 ; volt=0;

70

71 copy $3 \mathrm{~d}$ from=M1line8; to=M1line 7; direction=-1*M1pitch, 0,0 ; volt=0;

72

73 copy $3 \mathrm{~d}$ from=M1line7; to=M1line6; direction=-1*M1pitch, 0,$0 ;$ volt=0;

74

75 copy $3 \mathrm{~d}$ from=M1line6; to=M1line5; direction=-1*M1pitch, 0,0 ; volt=0;

76

77 copy $3 \mathrm{~d}$ from=M1line 5 ; to=M1line 4 ; direction=-1*M1pitch, 0,0 ; volt $=0$; 78

79 copy $3 \mathrm{~d}$ from=M1line4; to=M1line3; direction=-1*M1 pitch, 0,0 ; volt=0;

80

81 copy $3 \mathrm{~d}$ from=M1line3; to $=\mathrm{M} 1$ line2; direction=- $1 * \mathrm{M} 1$ pitch, 0,$0 ;$ volt $=0$;

82

83 copy $3 \mathrm{~d}$ from=M1line2; to $=\mathrm{M} 1$ line 1 ; direction= $1 * \mathrm{M} 1$ pitch, 0,0 ; volt $=0$;

84

85 block name=M2line3; width $=X$ total; length=M2width; volt $=0$;

$86 \mathrm{v1}=0,1.5^{*}$ M2pitch,Sthick+ILD0thick+M1 thick+ILD1 thick;

87 v2 $=0,1.5 *$ M2pitch,Sthick+ILD0thick+M1thick+ILD1thick+M2thick;

88

89 copy $3 \mathrm{~d}$ from=M2line 3 ; to $=$ M2line 2 ; direction=0, $-1 *$ M2pitch, 0 ; volt $=1$;

90

91 copy $3 \mathrm{~d}$ from=M2line2; to $=\mathrm{M} 2$ line 1 ; direction=0,-1*M2pitch, 0 ; volt $=0$;

92

93 block name $=$ Substrate; width $=$ Xtotal; length $=$ Ytotal; volt $=0$;

$94 \mathrm{vl}=0,0,0$;

95 v2 $=0,0$, Sthick;

96

97 window $3 \mathrm{~d}$ v $1=-0.5 *$ Xtotal, $-0.5 *$ Y total, 0.0 ;

98 v2 $=0.5 *$ Xtotal, $0.5 *$ Ytotal,Ztotal;

99

100 options set_grid $=500000$;

101

102 potential

*** POTENTIAL CALCULATION [Coulombs]

Charge on M1line $10=-8.337879 \mathrm{e}-17$

Charge on M1line9 $=-8.476684 \mathrm{e}-17$

Charge on M1line8 $=-8.471164 \mathrm{e}-17$

Charge on M1line7 $=-8.482564 \mathrm{e}-17$

Charge on M1line6 $=-8.438169 \mathrm{e}-17$

Charge on M1line $5=-8.432124 \mathrm{e}-17$

Charge on M1line4 $=-8.441266 \mathrm{e}-17$ 
Charge on M1line3 = -8.46493 le-17

Charge on M1line2 = $-8.582391 \mathrm{e}-17$

Charge on M1line1 = $-8.443481 \mathrm{e}-17$

Charge on M2line $3=-5.231111 \mathrm{e}-16$

Charge on M2line2 $=1.905325 \mathrm{e}-15$

Charge on M2line1 $=-5.206713 \mathrm{e}-16$

Charge on Substrate $=-1.609720 \mathrm{e}-17$

\section{REFERENCES:}

[1] Open SIPPs International " Proposal for SIPPs, Standard Interconnect Performance Parameters", California, USA, Sep. 1998

[2] SI2 "Silicon Integration Initiative", World-wide-web site http://www'si2.org., Texas, USA, 1998

[3] Raphael ${ }^{\mathrm{TM}}$ v4.0, TMA Inc, Sunnyvale, CA, USA, 1997

[4] QuickCap ${ }^{\mathrm{TM}}$ v1.1, RLC, Fairfax, VA, USA, 1996

[5] A.Chou, et al., "Characterization and Application of Interconnect Process Parameters", Dig. ICMTS, Japan, Mar. 1998.

[6] L.-F. Chang, et al., "Incorporating process induced effects into RC extraction", Dig. VLSI 99, India, January 1999. 